\title{
Prevalence of hearing loss in a population of schoolchildren with Down syndrome from Bogotá, Colombia
}

\author{
Iván Pradilla, M.D. ${ }^{a}$, Andrés Sarmiento-Buitrago, Student ${ }^{b}$, \\ Irma Carvajalino-Monje, Audiologist ${ }^{c}$, Alberto Vélez-Van-Meerbeke, M.D. ${ }^{a}$ and \\ Claudia Talero-Gutiérrez, M.D. ${ }^{a}$
}

\begin{abstract}
Introduction: Children with Down syndrome have a higher prevalence of hearing disorders, secondary to anatomical and physiological variations. No universal hearing screening is implemented in Colombia, so there are no data available on the prevalence of hearing loss in this population. The objective of this study was to determine the frequency and type of such disordersin this population based on hearing tests. Patients and methods: This was a cross-sectional study conducted at two institutions specialized in accompanying and supporting the families of children with Down syndrome in Bogotá, D.C., Colombia. Between October 2017 and September 2018, a behavioral and / or pure-tone audiometry and an impedance audiometry were done in 40 and 37 children respectively. Subjects attended the institutions twice a week and were integrated at school, up to fifth grade. Prevalence was estimated and characteristics, clinical history, and language developmental milestones were described.

Results: The sample was made up of 40 children (22 girls) aged 6-18 years (mean: 11.23). A history of otitis was found in 19 children and of mild to moderate hearing loss, in 17 , predominately conductive. A delay in language developmental milestones was observed, apparently not associated with hearing loss.

Conclusions: A sample of children with Down syndrome from Bogotá showed a high prevalence of hearing loss, consistent with the results of previous studies. These data stress the need for a strict control of hearing status based on standardized parameters and guidelines.

Key words: Down syndrome, hearing loss, universal hearing screening.
\end{abstract}

http: / / dx.doi.org/10.5546/ aap.2020.eng.e1

To cite: Pradilla I, Sarmiento-Buitrago A, CarvajalinoMonje I, Vélez-Van-Meerbeke A, Talero-Gutiérrez C. Prevalence of hearing loss in a population of school children with Down syndrome from Bogotá, Colombia. Arch Argent Pediatr 2020;118(1):e1-e7.

\section{INTRODUCTION}

Down syndrome (DS) is the most common aneuploidy; its prevalence has been estimated to be 8.3-13.8 per every 100000 live births in the United States. ${ }^{1,2}$ Multiple studies have associated DS with various heart, hematological, gastrointestinal, neurological, and ear, nose and throat (ENT) comorbidities that affect its morbidity and mortality. ${ }^{3,4}$ The most common ENT comorbidities include hearing and upper respiratory disorders and obstructive sleep apnea syndrome. ${ }^{3}$

Among hearing disorders, conductive, sensorineural, and mixed hearing loss are more common in children with DS and may be observed at birth or develop at a later stage..$^{5-7}$ They are associated with anatomical abnormalities of the ear, cerumen impaction, otitis media with effusion (OME) or cholesteatoma. ${ }^{8}$ Ossicular chain abnormalities and incudomalleolar joint deformity are also common. ${ }^{9}$ Likewise, Eustachian tube dysfunction has also been observed, which is the result of variations in craniofacial structures and hypotonia. ${ }^{3,10}$ In DS patients, the peak prevalence of OME is more than $60 \%$ at 6-7 years old, greater than in the general population. ${ }^{11,12}$ The presence of OME in children with DS is related to worse hearing test results. ${ }^{10}$ The prevalence of hearing loss has been established at $35 \%$ in different populations. ${ }^{13,14}$

Hearing disorders in the early stages of life have a negative impact on the development of speech and vocabulary. ${ }^{15}$ In addition, subjects with DS have a slower language development and poorer auditory 
memory and grammar comprehension performance. $^{16}$

In our setting, hearing screenings are not implemented universally and the follow-up and control of high-risk populations, as is the case of DS, is not sufficient. ${ }^{17}$ Therefore, there is not enough information about the prevalence of hearing disorders in the population of schoolchildren with DS.

\section{OBJECTIVE}

The objective of this study was to determine the frequency and type of hearing disorders in a group of school-aged children with DS.

\section{METHODS}

For this cross-sectional study, the eligible population corresponded to 300 schoolchildren aged 6-18 years attending therapy sessions at Corporación Síndrome de Down and Fundación Fe twice a week. The rest of the time, they were integrated at school (from preschool to fifth grade). These were public schools located in the city of Bogotá, where a regulation of the Ministry of Education has established the integration of children with different disabilities in regular schools.

Subjects were recruited between October 2017 and September 2018. For the invitation to participate in the study, the institutions posted signs and approached subjects personally. Interested parents signed a participation form and gave their informed consent after receiving a notice by one of the investigators.

\section{Inclusion, exclusion, and removal criteria}

All children with DS attending both institutions were eligible. The only exclusion criterion was children's inability to complete hearing tests due to understanding limitations or difficulty to follow directions, as reported by their parents and teachers.

\section{Procedures}

Once the informed consent form was signed, the subjects' case history was obtained, including demographic data, medical history and language development history of interest for this study. Children had an otoscopy done before hearing tests to determine their ear canal status and check for earwax blockage or other conditions preventing test performance. A sample of 40 children who met the criteria was established. Those who were not able to complete the tests due to a temporary disease (respiratory tract infection, earwax impaction) received follow-up. Children capable of giving their assent were informed by the investigator about the study activities and were assessed once they had agreed to participate. Three children had not given their assent for the impedance audiometry, so it was not performed on them.

The tests were scheduled to take place at the Foundation "Centro de Investigación e Información en Deficiencias Auditivas" (CINDA) at the parents' convenience. Tests were performed in a soundproof cabin in the presence of parents or a guardian, and children were given time to adapt and learn the task required of them. Tests included pure-tone, behavioral, and impedance audiometries and were carried out by speech therapists experienced in assessing the pediatric population. The equipment used was a Starkey AA30 audiometer and an Amplaid A766 impedance audiometer.

Hearing loss was diagnosed based on the World Health Organization's classification, ${ }^{18}$ defined according to the average thresholds in the $0.5 \mathrm{kHz}, 1 \mathrm{kHz}, 2 \mathrm{kHz}$, and $4 \mathrm{kHz}$ frequencies. This classification defined an average of 26-40 $\mathrm{dB}$ as mild; $41-60 \mathrm{~dB}$, as moderate; $61-80 \mathrm{~dB}$, as severe; and $>81 \mathrm{~dB}$, as profound.

In the impedance audiometer, curves were classified as type A, B or C. Type A tympanograms show a clear peak around atmospheric pressure and are common in subjects without hearing disorders. If a type A curve displays a shallow peak, it is classified as AS (common in otosclerosis or reduced compliance). If a type A curve displays a very sharp peak, it is classified as AD, which is observed in normal ears or those with flaccid eardrum or scarring. Type B curves are basically shallow across the pressure range and are typical in cases of effusion or fluid in the middle ear. However, a type B curve could also be seen in blockage of the ear canal (cerumen). A type $C$ tympanogram shows a negative peak pressure below $-100 \mathrm{daPa}$, indicating negative pressure in the middle ear. This is associated with Eustachian tube disorders and fluid in the middle ear. ${ }^{19}$

\section{Statistical analysis}

A sample size of 33 subjects was estimated considering a prevalence of $35 \%$, as previously reported by Austeng, with a $10 \%$ error and a $5 \% \alpha .^{13}$ 
The data collected from the case history, the physical examination, and the hearing tests were entered into a validated database. Data quality was checked periodically and five pieces of data were selected at random. All statistical analyses were performed using R (v3.4.2) software. ${ }^{20}$ Continuous variables were described as mean and standard deviation; discrete variables, as percentage. A summary audiometry curve was estimated considering the mean and confidence interval for each frequency, and the distribution of language developmental milestones was described. Based on the $35 \%$ prevalence results mentioned above, ${ }^{13}$ a binomial distribution was simulated to obtain the $90 \%$ and $50 \%$ quantiles. These values were used to develop an $a$ priori beta distribution, updated with the results of this study to establish a credible interval.

\section{Ethical aspects}

The study protocol was approved by the Research Ethics Committee of Universidad del Rosario, Bogotá, D.C. In addition, a Specific Research Agreement was signed to conduct this study at the participating institutions upon approval of the final protocol.

\section{RESULTS}

Out of the 300 eligible subjects who regularly attended both institutions, a sample of 74 interested families was obtained. Some participants did not attend the hearing tests or could not be contacted, so the final sample was made up of 40 subjects (Figure 1). Based on the information provided by the institutions, it was established that participants' age or sex were consistent with those of non-participating subjects. Behavioral/pure-tone audiometries were done in the 40 included children; whereas an impedance audiometry, in 37 of them. A sample of 40 children aged 6-18 years was obtained, with a similar number of males and females (22 girls) (Table 1).

According to their perinatal history, there was a high frequency of preterm birth and low birth weight (Table 2). Twenty-two subjects required some form of ventilatory support; and 20, phototherapy due to hyperbilirubinemia.

A total of 21 parents did not recall their child having otitis in the past. In addition, 8 reported 2 or more otitis episodes per year. Information about ototoxic drug use was unclear. Parents more commonly reported at least 1 upper respiratory tract infection episode $(n=30) ; 11$ of them mentioned $\geq 3$ episodes per year (Table 2 ).

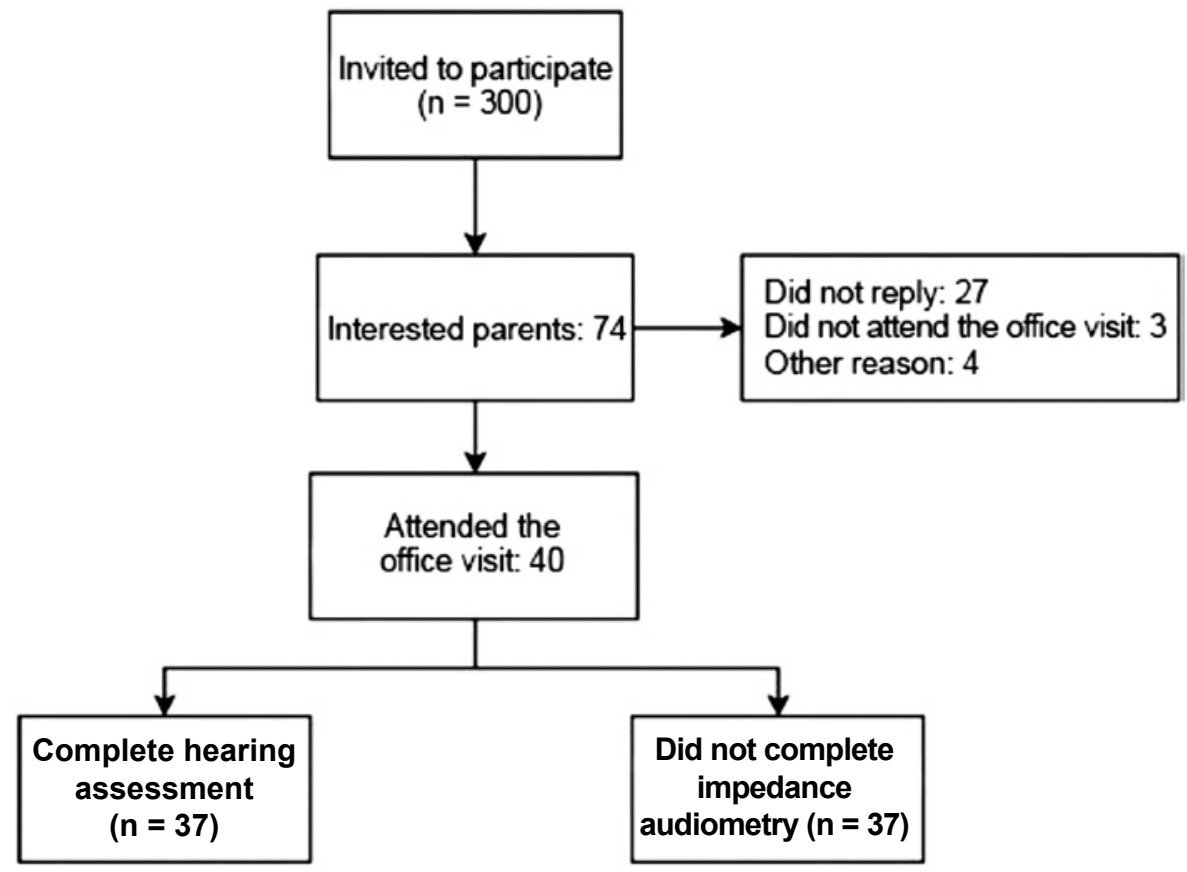


A significant percentage of children $(92.5 \%$, $\mathrm{n}=37$ ) had attended an audiology visit at different times while growing up. However, the type of assessment varied (auditory brainstem response, impedance audiometry and stapedial reflexes, and pure-tone or behavioral audiometry) (Table 3).

The physical examination found cerumen impaction in 11 children. Recommendations were made to these children or they were referred to the Ear, Nose and Throat Department, to enable subsequent audiological evaluation. No child showed external otitis signs.

As per hearing tests, the prevalence of any type of hearing loss was $42.5 \%(\mathrm{n}=17)$. The prevalence distribution showed a $95 \%$ credible interval of $27.8-45.3 \%$. The severity of hearing loss was mild in 13 subjects and moderate in 4 . Among those for whom it was possible to

TABLE 1. Sample characteristics $(N=40)$

\begin{tabular}{lc}
\hline Outcome measure & Mean (SD) \\
& $\%$ (n) \\
\hline Age & $11.23(3.21)$ \\
Sex (F) & $45(18)$ \\
Free trisomy 21 & $92.5(37)$ \\
Paternal age at birth & $38.0(8.41)$ \\
Maternal age at birth & $35.2(5.48)$ \\
\hline
\end{tabular}

SD: standard deviation.

TABLE 2. Perinatal and infectious history $(N=40)$

\begin{tabular}{lc}
\hline Outcome measure & $\begin{array}{c}\text { Mean (SD) } \\
\%(\mathbf{n})\end{array}$ \\
\hline Gestational age & $37(2.56)$ \\
Preterm birth & $25(10)$ \\
Birth weight $(\mathrm{g})$ & $2551(710)$ \\
Low birth weight & $32.5(13)$ \\
Birth length $(\mathrm{cm})$ & $47(4.84)$ \\
Infections during pregnancy & $15(6)$ \\
Phototherapy & $50(20)$ \\
Ventilatory support requirement & $55(22)$ \\
History of ARTI & \\
None & $25(10)$ \\
Sporadic & $27.5(11)$ \\
$1-2$ times a year & $20(8)$ \\
3 or more times a year & $27.5(11)$ \\
History of otitis & \\
None & $52.5(21)$ \\
Sporadic & $27.5(11)$ \\
$1-2$ times a year & $7.5(3)$ \\
3 or more times a year & $12.5(5)$ \\
\hline
\end{tabular}

SD: standard deviation; ARTI: acute respiratory tract infection. establish the type of hearing loss, 1 subject had sensorineural hearing loss; 12 , conductive hearing loss; and 1, mixed type.

It was not possible to perform an impedance audiometry in 3 children because they did not assent to it. Bilateral type A curves were observed in 11 subjects. Type $B$ curves were noted in one ear of 9 subjects; AS curves, in 6; AD curves, in 2; and $C$ curves, in 5 . Four additional curves showed negative pressure shifts. Among the latter, 2 had conductive hearing loss.

Figure 2 shows the mean results obtained in bilateral audiometries, together with their $95 \%$ confidence interval. Even in the case of bilateral hearing loss, it was observed to be minimal to mild, as per the American Speech-LanguageHearing Association classification (minimal: 16$25 \mathrm{~dB}$; mild: $26-40 \mathrm{~dB}$ ). ${ }^{21}$ Figure 3 shows language developmental milestones ${ }^{22}$ and their delayed attainment.

\section{DISCUSSION}

The prevalence of hearing loss in this population was $42.5 \%$, close to that found in previous reports of approximately $35 \% .{ }^{5,14}$ If compared to the overall prevalence of hearing disorders in Colombia, which is $17.3 \%$, the importance of providing special health care looking for a timely diagnosis is evident. ${ }^{23}$ The higher prevalence of hearing loss in DS has been associated with features typical of this condition..$^{5,24}$ The etiologies described in other studies showed that up to $75 \%$ of hearing loss cases are conductive..$^{24}$ Other studies suggested a high frequency of sensorineural hearing loss in people with DS, possibly in relation to the dysplastic characteristics of the inner ear. ${ }^{25,26}$ Nonetheless, in this study, only 1 subject had an audiometry result consistent with sensorineural hearing loss.

TABLe 3. Prior hearing tests $(N=40)$

\begin{tabular}{lc}
\hline Test & $\%$ (n) \\
\hline $\begin{array}{l}\text { None } \\
\text { Only behavioral/ }\end{array}$ & $7.5(3)$ \\
pure-tone audiometry & $32.5(13)$ \\
Auditory brainstem response & $17.5(7)$ \\
Behavioral/pure-tone audiometry & \\
+ impedance audiometry & $12.5(5)$ \\
Behavioral/pure-tone audiometry & \\
+ auditory brainstem response & $15(6)$ \\
All & $15(6)$ \\
\hline
\end{tabular}


The results of the impedance audiometry showed a high prevalence of type $B$ and $C$ curves. Previous studies had shown this sort of tendency and related it to the characteristics of children with DS, such as Eustachian tube dysfunction, ossicular chain abnormalities, and other middle ear alterations. ${ }^{27}$ Although type B curves were common, no clinical finding was indicative of acute otitis media (AOM), OME or other abnormality. These findings emphasized that structural alterations in the DS spectrum act as risk factors for conditions with a negative impact on hearing.

Children with DS have language development problems that may be related to hearing disorders. ${ }^{16,28}$ Chapman et al., suggested that these children have more language comprehension and auditory memory deficits

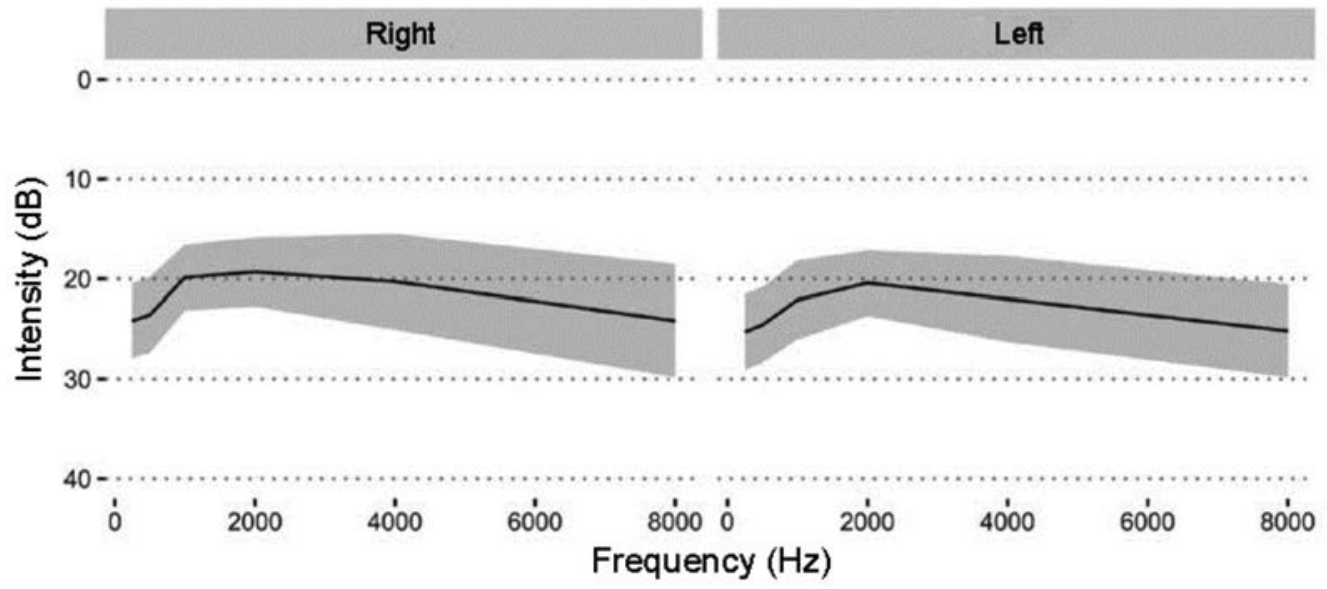

Mean and $95 \%$ confidence interval values (gray area) for each ear.

FigURE 3. Distribution of age at the time subjects reached language developmental milestones $(N=40)$

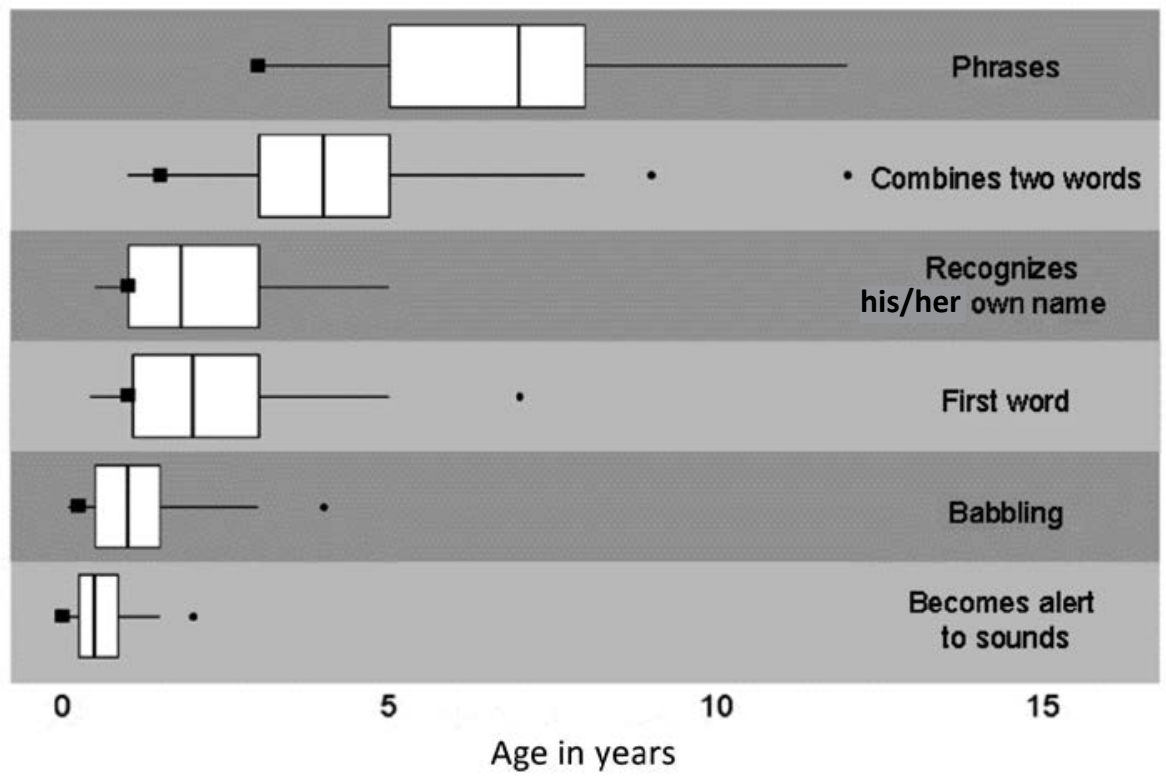

The age at the time of attainment of milestones in the standard population is shown as a black square. Being alert to sounds is expected at birth; babbling, at 3 months old; uttering the first word and recognizing their own name, at 1 year old; combining two words, at 18 months old; and forming phrases, at 3 years old. ${ }^{22}$ 
compared to the control group..$^{28}$

Audiometry results did not show an association with the age at the time subjects reached language milestones. According to the bibliography, children with DS show major language deficits, particularly in expressive vocabulary, grammar, and short-term verbal memory. ${ }^{29}$ However, current data are not enough to establish an association between hearing loss and this kind of finding. Although some studies suggested a relation between early hearing loss and language development disorders in DS, ${ }^{16}$ in our sample, hearing loss was minimal to mild and would not account for a language developmental delay.

A relevant frequency of medical and perinatal history was observed in this population. Recurrent AOM events during childhood and gestational infections have been associated with a higher incidence of hearing loss. ${ }^{18}$ This study supports the evidence on the high prevalence of ENT disorders in subjects with DS who have a frequent history of OME, AOM, earwax impaction, among others. ${ }^{8,30,31}$

Prior hearing test results varied greatly, which indicated that a protocol similar to that recommended by the Joint Committee on Infant Hearing $^{32}$ or the American Academy of Pediatrics ${ }^{33}$ had not been followed. However, $92.5 \%$ of children had at least one hearing test done, which served as guidance for treatment and management. This suggests that, although recommendations are not followed extensively, an effort has been made to detect hearing loss in this population in an early manner.

\section{Study limitations}

This was a descriptive, cross-sectional study, so it was possible to characterize the population but not to estimate the association among different outcome measures or provide follow-up over time. Although a large part of the population who was invited to participate was not recruited, no differences were observed in terms of the general characteristics of the study sample. However, we cannot rule out the fact that the sample was self-selected due to the interest of parents secondary to a greater or smaller perception of symptoms.

The population was obtained from two institutions for children with DS, so it may have certain special characteristics not present in the general population. Nonetheless, both institutions were referral facilities, making them interesting for an approach to the general population and paving the way for future studies.
Given that the information about children's development and history was not obtained from formal sources, it may include a recall bias. Such effect may be more significant in older children.

\section{CONCLUSIONS}

In this sample of schoolchildren with DS, the prevalence of hearing loss was $42.5 \%$, predominately, minimal to mild conductive hearing loss. Based on the severity of hearing loss observed, the delay in communication skills does not appear to be related to it and may be attributed to cognitive and developmental characteristics of children with DS. The high prevalence of conductive hearing loss underpins the need for control and follow-up in this population. Follow-up should adhere to the recommendations of international organizations and encourage parental compliance.

\section{REFERENCES}

1. Parker SE, Mai CT, Canfield MA, Rickard R, et al. Updated national birth prevalence estimates for selected birth defects in the United States, 2004-2006. Birth Defects Res A Clin Mol Teratol. 2010; 88(12):1008-16.

2. Presson AP, Partyka G, Jensen KM, Devine OJ, et al. Current Estimate of Down Syndrome Population Prevalence in the United States. J Pediatr. 2013;163(4):1163-8.

3. Kanamori G, Witter M, Brown J, Williams-Smith L. Otolaryngologic Manifestations of Down Syndrome. Otolaryngol Clin North Am. 2000;33(6):1285-92.

4. Roizen NJ. Down syndrome: Progress in research. Ment Retard Dev Disabil Res Rev. 2001; 7(1):38-44.

5. Park AH, Wilson MA, Stevens PT, Harward R, et al. Identification of Hearing Loss in Pediatric Patients with Down Syndrome. Otolaryngol Head Neck Surg. 2012; 146(1):135-40.

6. Tedeschi AS, Roizen NJ, Taylor HG, Murray G, et al. The prevalence of congenital hearing loss in neonates with Down syndrome. J Pediatr. 2015;166(1):168-1.

7. Nightengale E, Yoon P, Wolter-Warmerdam K, Daniels D, et al. Understanding Hearing and Hearing Loss in Children With Down Syndrome. Am J Audiol. 2017; 26(3):301-8.

8. Díaz-Galindo L, Talero-Gutierrez C, Pinilla- Zuleta N, Sánchez-Cruz A, et al. Alteraciones auditivas y desarrollo del lenguaje en niños con síndrome de Down: revisión sistemática de la literatura. Acta Pediatr Esp. 2018; 76(12):e1-8.

9. Fausch C, Röösli C. The incudomalleolar articulation in Down syndrome (trisomy 21): a temporal bone study. Otol Neurotol. 2015; 36(2):348-53.

10. Austeng ME, AkreH, Øverland B, Abdelnoor M, et al. Otitis media with effusion in children with Down syndrome. Int J Pediatr Otorhinolaryngol. 2013; 77(8):1329-32.

11. Maris M, Wojciechowski M, Van de Heyning P, Boudewyns A. A cross-sectional analysis of otitis media with effusion in children with Down syndrome. Eur J Pediatr. 2014; 173(10):1319-25.

12. Rosenfeld RM,Shin JJ,SchwartzSR, Coggins R, et al. Clinical Practice Guideline: Otitis Media with Effusion (Update). Otolaryngol Head Neck Surg. 2016; 154(1 Suppl):S1-41. 
13. Austeng ME, Akre H, Falkenberg E-S, Øverland B, et al. Hearing level in children with Down syndrome at the age of eight. Res Dev Disabil. 2013; 34(7):2251-6.

14. Lau WL, Ko CH, Cheng WW. Prevalence and Parental Awareness of Hearing Loss in Children with Down Syndrome. Chin Med J (Engl). 2015; 128(8):1091-5.

15. Barker DH, Quittner AL, Fink NE, Eisenberg LS, et al. Predicting behavior problems in deaf and hearing children: the influences of language, attention, and parent-child communication. Dev Psychopathol. 2009; 21(2):373-92.

16. Laws G, Hall A. Early hearing loss and language abilities in children with Down syndrome. Int I Lang Commun Disord. 2014; 49(3):333-42.

17. Rojas-Godoy AL, Gómez-Gómez O, Rivas-Muñoz FA. Cumplimiento de la normatividad vigente para la detección temprana de la hipoacusia neonatal. Rev Salud Publica. 2014; 16(3):462-72.

18. Díaz C, Goyco o lea M, Cardemil F. Hipoacusia: Trascendencia, incidencia y prevalencia. Rev Med Clin Condes. 2016; 27(6):731-9.

19. Gelfand SA. Acoustic Immitance Assessment. In Gelfand SA. Essentials of audiology. $4^{\text {th }}$ ed. New York: Thieme; 2016.P.187.

20. Team RC. R: a language and environment for statistical computing. Vienna, Austria: R Foundation for Statistical Computing; 2018. [Accessed on: July 2019]. Available at: https:/ / www.R-project.org.

21. Clark JG. Uses and abuses of hearing loss classification. ASHA. 1981; 23(7):493-500.

22. Owens RE. Desarrollo del lenguaje. 5. ${ }^{\text {ta }}$ ed. Madrid:Pearson Educación; 2003.

23. Departamento Administrativo Nacional de Estadística. Censo General 2005. Discapacidad. Personas con limitaciones permanentes. DANE; 2006. [Accessed on: July 2019]. Available at: https: / / www.dane.gov.co / files / censo2005/discapacidad.pdf.
24. Shott SR. Down syndrome: Common otolaryngologic manifestations. Am J Med Genet C Semin Med Genet. 2006; 142C(3):131-40.

25. Clark CM, Patel HH, Kanekar SG, Isildak H. Enlarged vestibular aqueducts and other inner-ear abnormalities in patients with Down syndrome. J Laryngol Otol. 2017; 131(4):298-302.

26. Intrapiromkul J, Aygun N, Tunkel DE, Carone M, et al. Inner ear anomalies seen on CT images in people with Down syndrome. Pediatr Radiol. 2012; 42(12):1449-55.

27. Satwant S, Subramaniam KN, Prepageran N, Raman R, et al. Otological disorders in Down's Syndrome. Med J Malaysia. 2002; 57(3):278-82.

28. ChapmanR. LanguagelearninginDownsyndrome:Thespeech and language profile compared to adolescents with cognitive impairment of unknown origin. Downs Syndr Res Pract. 2006; 10(2):61-6.

29. NæssKAB,LysterSAH,HulmeC,Melby-LervågM.Language and verbal short-term memory skills in children with Down syndrome: a meta-analytic review. Res Dev Disabil. 2011; 32(6):2225-34

30. Bassett EC, Musso MF. Otolaryngologic management of Down syndrome patients: what is new? Curr Opin Otolaryngol Head Neck Surg. 2017; 25(6):493-7.

31. Bull MJ. Health Supervision for Children With Down Syndrome. Pediatrics. 2011; 128(2):393-406.

32. American Academy of Pediatrics, Joint Committee on Infant Hearing. Year 2007 Position Statement: Principles and Guidelines for Early Hearing Detection and Intervention Programs. Pediatrics. 2007; 120(4):898-921.

33. Ivan DL, Cromwell P. Clinical Practice Guidelines for Management of Children With Down Syndrome: Part II. J Pediatr Health Care. 2014; 28(3):280-4. 